\title{
Prognostic significance of MEOX2 in gliomas
}

\author{
Gaelle Tachon $\mathbb{1}^{1,2,3} \cdot$ Konstantin Masliantsev $\mathbb{1}^{1,2,3} \cdot$ Pierre Rivet $^{3} \cdot$ Christos Petropoulos $^{1,2,3} \cdot$ Julie Godet $^{4}$. \\ Serge Milin ${ }^{4}$ Michel Wager ${ }^{1,2,5} \cdot$ Pierre-Olivier Guichet $\mathbb{I}^{1,2,3} \cdot$ Lucie Karayan-Tapon ${ }^{1,2,3}$
}

Received: 13 July 2018 / Revised: 20 November 2018 / Accepted: 21 November 2018 / Published online: 18 January 2019

(c) United States \& Canadian Academy of Pathology 2019

\begin{abstract}
Gliomas are the most common malignant primary tumors in the central nervous system and have variable predictive clinical courses. Glioblastoma, the most aggressive form of glioma, is a complex disease with unsatisfactory therapeutic solutions and a very poor prognosis. Some processes at stake in gliomagenesis have been discovered but little is known about the role of homeobox genes, even though they are highly expressed in gliomas, particularly in glioblastoma. Among them, the transcription factor Mesenchyme Homeobox 2 (MEOX2) had previously been associated with malignant progression and clinical prognosis in lung cancer and hepatocarcinoma but never studied in glioma. The aim of our study was to investigate the clinical significance of MEOX2 in gliomas. We assessed the expression of MEOX2 according to IDH1/2 molecular profile and patient survival among three different public datasets: The Cancer Genome Atlas (TCGA), The Chinese Glioma Genome Atlas (CGGA) and the US National Cancer Institute Repository for Molecular Brain Neoplasia Data (Rembrandt). We then evaluated the prognostic significance of MEOX2 protein expression on 112 glioma clinical samples including; 56 IDH1 wildtype glioblastomas, 7 IDHI wild-type lower grade gliomas, 49 IDH1 mutated lower grade gliomas. Survival rates were estimated by the Kaplan-Meier method followed by uni/multivariate analyses. We demonstrated that MEOX2 was one of the transcription factors most closely associated with overall survival in glioma. Moreover, MEOX2 expression was associated with $I D H 1 / 2$ wildtype molecular subtype and was significantly correlated with overall survival of all gliomas and, more interestingly, in lower grade glioma. To conclude, our results may be the first to provide insight into the clinical significance of MEOX2 in gliomas, which is a factor closely related to patient outcome. MEOX2 could constitute an interesting prognostic biomarker, especially for lower grade glioma.
\end{abstract}

\section{Introduction}

The prediction of clinical behavior, response to therapy, and outcome of glioma is challenging. Despite the past 25 years of research into glioma biology, leading to the discovery of

These authors contributed equally: Pierre-Olivier Guichet, Lucie Karayan-Tapon.

Supplementary information The online version of this article (https:// doi.org/10.1038/s41379-018-0192-6) contains supplementary material, which is available to authorized users.

Pierre-Olivier Guichet

pierre-olivier.guichet@chu-poitiers.fr

$\triangle$ Lucie Karayan-Tapon

lucie.karayan-tapon@chu-poitiers.fr

1 Inserm U1084, Laboratoire de Neurosciences Expérimentales et Cliniques, Poitiers F-86073, France several molecular alterations in lower grade and high grade gliomas, therapy development for adult diffuse glioma remains uneven. Glioblastoma remains the most common and aggressive primary brain tumor with a very poor prognosis with 5-year overall survival rate below 5\% [1]. Current treatment involves surgery followed by radiation and temozolomide chemotherapy but tumor recurrence appears inevitable [2]. Classification of gliomas had traditionally been based on histologic features and degrees of malignancy after hematoxylin and eosin staining criteria according to the World Health Organization (WHO) 2007 classification [3]. But this classification has presented

2 Université de Poitiers, F-86073 Poitiers, France

3 CHU de Poitiers, Laboratoire de Cancérologie Biologique, Poitiers F-86022, France

4 CHU de Poitiers, Service d'Anatomo-Cytopathologie, Poitiers F86021, France

5 CHU de Poitiers, Service de Neurochirurgie, Poitiers F-86021, France 
several shortcomings, particularly major interobserver variability in histological interpretation [4, 5]. More accurate and reproducible criteria were urgently needed to reliably discriminate glioma subtypes and better predict patient outcome. To serve this purpose the revised 2016 WHO classification of tumors of the central nervous system combines histological and molecular features and places molecular biomarkers, such as the isocitrate dehydrogenase 1 and $2(I D H 1 / 2)$ mutations and the co-deletion of chromosome arms $1 p$ and $19 q$, in stages [6]. Despite an extended time of molecular investigation of glioma profile, some interesting molecular biomarkers may have been missed, including MEOX2, to the discovery of which the current study is largely devoted.

Mesenchyme Homeobox 2 (MEOX2, also called GAX) belongs to the homeobox gene family and has been established as a growth arrest specific homeobox by cycline dependant kinase inhibitor p21 and p16 activation [7]. It is expressed in vascular smooth muscle and vascular endothelial cells $[8,9]$. According to a recently published study, MEOX2 overexpression suppresses proliferation and migration of vascular smooth muscle cells [10]. In addition, $M E O X 2$ expression could inhibit endothelial cell proliferation and angiogenesis by $N F-k B$ down-regulation [11]. In a tumoral context, the dual role of MEOX2 has been reported. In hepatocellular carcinoma and in larynx carcinoma, reduced $M E O X 2$ expression has been significantly correlated with short overall survival (OS), whereas in lung cancer, MEOX2 overexpression has been correlated with chemoresistance [12-14]. It has also been described as a potential tumor suppressor gene in Wilms tumor [15]. Another study showed that MEOX2-GLI1 axis was involved in cell viability, cellular proliferation and migration capacity in lung cancer, as well as associated with overall clinical survival and therapy prognosis [16].

MEOX2 gene is located at $7 \mathrm{p} 21.2$ locus and gain of chromosome 7 is part of the molecular signature of glioblastoma, especially the classic Verhaak subtype [6, 17]. Another study describes a cohort of 117 mesenchymal glioblastomas and identified a high risk signature of 17 genes comprising MEOX2 which correlated with overall survival [18]. Another work reported MEOX2 downregulation in 15 pools of various cells (glioma stem cell line, astrocytes overexpressing oncogenic and iPSCinducing factors, conventional glioblastoma cell lines) compared to normal astrocytes [19]. But MEOX2 expression has not been evaluated in a combined cohort of lower grade/high grade gliomas or according to the WHO 2016 classification. Moreover, its expression in glioma clinical samples and its role in gliomagenesis have never been deciphered.

The aim of our study was to assess MEOX2 expression and its prognostic value in gliomas. We showed that
MEOX2 was an interesting prognostic marker in gliomas using a clinical cohort of 112 gliomas and public datasets, namely, The Cancer Genome Atlas (TCGA), The Chinese Glioma Genome Atlas (CGGA) and the US National Cancer Institute Repository for Molecular Brain Neoplasia Data (Rembrandt).

\section{Materials and Methods}

\section{Patient cohort, clinical data and molecular traits}

A total of 112 patients, operated at the University Hospital of Poitiers with de novo gliomas/glioblastomas diagnosed, were included in this study. The use of human tissue was granted by the secretary of state for education and research, directorate-general for research and innovation, bioethics unit (DC-2008-565), and in accordance with the Declaration of Helsinki. Median age at diagnosis was 51 years and the median survival was 25 months. Patients had received chemotherapy with temozolomide and radiotherapy in 56\% of cases (Table 1).

\section{Bioinformatic analyses}

Normalized RSEM gene-level RNAseq data, methylation profile (beta value) and Gistic2 thresholded copy number calls of glioblastoma and lower grade glioma cohort from TCGA were downloaded from Broad GDAC Firehose (gdac.broadinstitue.org). Clinical data were obtained from Table S1 of TCGA publication "Molecular Profiling Reveals Biologically Discrete Subsets and Pathways of Progression in Diffuse Glioma" (http://www.sciencedirect. com/science/article/pii/S009286741501692X). Normalized FPKM RNAseq data from the Chinese Glioma Genome Atlas (CGGA) were obtained from http://cgga.org.cn:9091/ gliomasdb/download.jsp. The level of MEOX2 mRNA was classified as low or high according to the mean. Lower grade gliomas $I D H I / 2$ wildtype subgroup was additionally classified according to quartiles. It has been acknowledged that primary and secondary glioblastomas are distinct tumor entities with distinct molecular features that originate from different precursor cells [20]. Hence, only $I D H I$ wild-type glioblastomas were taken into account in this study (for CGGA, TCGA and clinical datasets), as IDHI mutated glioblatomas are mostly secondary glioblastomas [21]. Microarray data from the US National Cancer Institute Repository for Molecular Brain Neoplasia Data (REMBRANDT) cohort were acquired from Gene Expression Omnibus (https://www.ncbi.nlm.nih.gov/geo/query/a cc.cgi?acc $=$ GSE68848), normalized by RMA and using a custom CDF downloaded from BrainArray (http://brainarra y.mbni.med.umich.edu/Brainarray/Database/CustomCDF/ 
Table 1 Clinical and molecular traits of patients from clinical cohort

\begin{tabular}{|c|c|c|c|c|}
\hline & & All gliomas & Lower Grade gliomas & Glioblastomas \\
\hline \multicolumn{2}{|l|}{ Number of patients (\%) } & $112(100)$ & $56(100)$ & $56(100)$ \\
\hline \multirow[t]{2}{*}{ Gender } & Female $(\%)$ & $44(39)$ & $24(43)$ & $20(36)$ \\
\hline & Male (\%) & $73(61)$ & $32(57)$ & $36(64)$ \\
\hline \multirow[t]{2}{*}{ Age } & Median (years) & 51 & 40 & 62 \\
\hline & (Min - Max) & $(22-83)$ & $(22-81)$ & $(32-83)$ \\
\hline \multirow[t]{2}{*}{ Resection type } & Biopsy (\%) & $22(20)$ & $9(16)$ & $13(23)$ \\
\hline & Resection (\%) & $90(80)$ & $47(84)$ & $43(77)$ \\
\hline \multirow[t]{3}{*}{ Treatment $(n=111)$} & Chemo/Radiotherapy (\%) & $62(56)$ & $6(11)$ & $56(100)$ \\
\hline & Chemotherapy (\%) & $6(5)$ & $6(11)$ & 0 \\
\hline & No $(\%)$ & $43(39)$ & $43(78)$ & 0 \\
\hline Survival & Median (Months) & 25 & 44 & 15 \\
\hline \multirow[t]{2}{*}{ Status } & Dead $(\%)$ & $71(63)$ & $15(27)$ & $56(100)$ \\
\hline & Alive $(\%)$ & $41(37)$ & $43(73)$ & $0(0)$ \\
\hline \multirow[t]{2}{*}{ Progression } & cases $(\%)$ & $72(64)$ & $18(32)$ & $54(96)$ \\
\hline & Median (Months) & 8 & 26 & 8 \\
\hline \multirow[t]{3}{*}{ Molecular traits } & IDH1 wildtype (\%) & $63(56)$ & $7(12)$ & $56(100)$ \\
\hline & $\begin{array}{l}\text { IDH1 mutated non- } \\
\text { codeleted }(\%)\end{array}$ & $25(22)$ & $25(45)$ & NA \\
\hline & $\begin{array}{l}\text { IDH1 mutated with } 1 \mathrm{p} / 19 \mathrm{q} \\
\text { codeletion }(\%)\end{array}$ & $24(21)$ & $24(43)$ & NA \\
\hline
\end{tabular}

CDF_download.asp). Z-score normalized expression values of MEOX2 were taken for the analysis of Ivy Glioblastoma Atlas Project with Allen Institute for Brain Science data sets (http://glioblastoma.alleninstitute.org/static/download.html). RNAseq data of glioblastoma and lower grade glioma cohort from TCGA were analyzed for enrichment of functional gene sets using Gene Set Enrichment Analysis (GSEA, https:// software.broadinstitute.org/gsea/index.jsp). C5.bp.v6.1 gene set comprising 4436 GO terms was extracted from the Molecular Signatures Database (MSigDB) in GSEAcompatible GMT format. Only the four most illustrative signatures with Normalized Enrichment Score $\geq 2.74$ and a False Discovery Rate $<0.001$ were disclosed. MEOX2 expression network according to TCGA data was performed using RTN and visualized using RedeR and igraph $\mathrm{R}$ package installed from Bioconductor (https://www.bioconductor.org/) or CRAN repository (https://cran.r-project.org/web/packages/). Positively regulated genes were illustrated in red, and negatively regulated genes in blue. The length between $M E O X 2$ and other genes symbolized the strength of the regulation.

\section{Immunohistochemical staining}

Tissue micro arrays were established using paraffinembedded tissue samples (10\% neutral buffered) from tumor biopsies or surgical removal of 112 glioma of patients. To overcome tumor heterogeneity, at least 3 biopsy cores of $1 \mathrm{~mm}$ diameter and $3 \mu \mathrm{m}$ thick were included in the recipient block using a tissue microarray workstation (Alphelys, France). For each case, a minimum of 3 cores were transferred from the selected areas to the recipient block, using a tissue microarray workstation (Alphelys, France).

Anti-MEOX2 polyclonal antibody produced in rabbit, from prestige antibodies powered by atlas antibodies, was chosen for immunohistochemistry experiments (SigmaAldrich, Missouri, USA). Immunohistochemistry was carried out manually using anti-MEOX2 antibody at 1/500 dilution. Slides were deparaffinized and heated in sodium citrate pH6 solution during $40 \mathrm{~min}$ at $96^{\circ} \mathrm{C}$ for antigenic retrieval. MEOX2 antibody was incubated overnight at $4{ }^{\circ} \mathrm{C}$ and displayed using the streptavidin-biotin-peroxidase method with diaminobenzidine as chromogen (Vectastain ${ }^{\circledR}$ ABC-HRP Kit, Vector laboratories, California, USA). The antibody was validated for immunohistochemistry analysis on placenta sample $(39+6$ months of amenorrhea) and chorionic villi presented the same endothelial cell staining as illustrated in human protein atlas (Figure S1a). Scoring of antibody staining for glioma samples was evaluated by a junior and a senior pathologist independently. Scoring of intensity staining was determined as follows: 0 , negative; 1 , weak; 2, moderate; and 3, strong (Figure S1b). MEOX2 positive score was settled at score $\geq 1$, while negative score at score $<1$.

\section{Molecular characterization}

Mutations in IDHI (R132H, R132C, R132S, R132G) were assessed by Sanger sequencing on a 3500Dx DNA 
Sequencer (Applied Biosystems) using the following primers Forward 5-CAGAGAAGCCATTATCTGC-3 and Reverse 5-GGAAATTTCTGGGCCATG-3. The PCR template comprised a denature step lasting $5 \mathrm{mins}$ at $95^{\circ} \mathrm{C}$ followed by 35 amplification cycles of $95^{\circ} \mathrm{C}$ for $45 \mathrm{~s}, 56^{\circ} \mathrm{C}$ for $45 \mathrm{~s}, 72{ }^{\circ} \mathrm{C}$ for $45 \mathrm{~s}$, and a final extension step at $72{ }^{\circ} \mathrm{C}$ for 7 mins. Loss of heterozigosity was evaluated by microsatellite analysis. To analyze the loss of heterozigosity at $1 \mathrm{p} 36$ and $19 \mathrm{q} 13$ loci, four microsatellite markers on $1 \mathrm{p} 36$ (D1S243, D1S199, D1S2734, D1S508) and four microsatellite markers on 19q13 (D19S112, D19S206, D19S412 and D19S596) were used. DNA was amplified and aliquots of the PCR reactions were subjected to electrophoresis and collected data were analyzed with genemapper software (Applied Biosystems). The loss of heterozigosity index was determined with the formula (height (A2/A1) control $\left./(\mathrm{A} 2 / \mathrm{A} 1)_{\text {tumoral }}\right)$. Index $<0.5$ and $>1.5$ was considered positive.

\section{Statistical analysis}

Descriptive statistics of the results were calculated with GraphPad Prism 6 (California, USA). SPSS (IBM, New York) software was used for univariate and multivariates analyses. Statistical significance was evaluated by KruskalWallis, Mann-Whitney, Fisher exact test, Mantel-Cox log rank or Cox regression tests $(* \mathrm{p}<0.05 ; * * \mathrm{p}<0.01$; *** $\mathrm{p}<$ $0.001)$. Cox proportional hazard regression model analysis on TCGA dataset was performed using BRB-ArrayTools (NIH). Survival rates were estimated by the Kaplan-Meier method.

\section{Results}

\section{MEOX2 is one of the transcription factors most associated with overall survival}

In order to identify new transcription factors associated with the overall survival in gliomas, we performed a Cox proportional hazard analysis on glioblastoma and lower grade glioma RNAseq data from TCGA. Surprisingly, we found that the top ten transcription factor genes encode homeobox proteins (Table 2). We focused our attention on $M E O X 2$ for which mRNA level was undeniably higher in gliomas than in other human tumors (Figure S2).

\section{MEOX2 is associated with glioma aggressiveness according to TCGA database}

The glioblastoma and lower grade glioma cohort comprised 134 glioblastomas (Grade IV) and 455 lower grade gliomas (214 Grade II and 241 Grade III). We showed that MEOX2 mRNA was more abundant in WHO Grade IV than in Grades II and III ( $\mathrm{p}<0.0001$, Fig. 1a). Moreover, MEOX2 mRNA was enriched in glioblastoma in comparison with other pathological subsets, namely oligodendroglioma, oligoastrocytoma and astrocytoma ( $<<0.0001$, Fig. 1b). More interestingly, we compared the MEOX2 mRNA level between molecular profiles and found that $I D H 1 / 2$ wildtype gliomas (lower grade glioma and glioblastoma) highly expressed $M E O X 2$ in comparison with IDH1/2 mutant gliomas $(n=648, p<0.0001$, Fig. 1c), independently of $1 \mathrm{p} 19 \mathrm{q}$ codeletion status. To gain better insight on how this higher MEOX2 mRNA abundance in IDH1/2 wildtype tumors could be explained, we analyzed copy number alteration of $M E O X 2$ and chr7 as well as methylation status of $M E O X 2$. As shown in Fig. 1d, amplification of $M E O X 2$ is strongly associated with chr7 gain. Moreover, a positive correlation was established between MEOX2 amplification and mRNA level $(r=0.3287, p<0.001)$ (Fig. 1d and S3A). On the contrary, a negative correlation was found between MEOX2 mRNA abundance and methylation profile $(\mathrm{r}=$ $-0.5339, \mathrm{p}<0.001$ ) (Fig. 1d and S3B). Analysis of KaplanMeier survival curves of the all glioma cohort showed that a high level of MEOX2 mRNA was associated with significantly shorter overall survival (14 months versus 80 months, $\mathrm{p}<0.0001$ ) (Fig. 1e). On the other hand, and
Table 2 The 10 most critical transcription factors highly correlated with patient overall survival in TCGA dataset (Cox proportional hazard analysis)

\begin{tabular}{lllllll}
\hline $\begin{array}{l}\text { Rank in Trancription } \\
\text { Factor list }\end{array}$ & $\begin{array}{l}\text { Rank in } \\
\text { gene list }\end{array}$ & Symbol & p-value & $\begin{array}{l}\text { False discovery } \\
\text { rate }\end{array}$ & $\begin{array}{l}\text { Hazard } \\
\text { Ratio }\end{array}$ & $\begin{array}{l}\text { Standard } \\
\text { deviation }\end{array}$ \\
\hline 1 & 27 & HOXA5 & $<1 \mathrm{e}-07$ & $<1 \mathrm{e}-07$ & 1.421 & 2.996 \\
2 & 33 & HOXD11 & $<1 \mathrm{e}-07$ & $<1 \mathrm{e}-07$ & 1.413 & 2.872 \\
3 & 49 & HOXA3 & $<1 \mathrm{e}-07$ & $<1 \mathrm{e}-07$ & 1.407 & 3.051 \\
4 & 59 & HOXA1 & $<1 \mathrm{e}-07$ & $<1 \mathrm{e}-07$ & 1.636 & 2.244 \\
5 & 86 & HOXB3 & $<1 \mathrm{e}-07$ & $<1 \mathrm{e}-07$ & 1.348 & 3.241 \\
6 & 97 & SHOX2 & $<1 \mathrm{e}-07$ & $<1 \mathrm{e}-07$ & 1.366 & 3.503 \\
7 & 111 & HOXD10 & $<1 \mathrm{e}-07<1 \mathrm{e}-07$ & 1.362 & 3.007 \\
8 & 123 & HOXA4 & $<1 \mathrm{e}-07$ & $<1 \mathrm{e}-07$ & 1.357 & 3.19 \\
$\mathbf{9}$ & $\mathbf{1 3 2}$ & MEOX2 & $<\mathbf{1 e - 0 7}<\mathbf{1 e - 0 7}$ & $\mathbf{1 . 3 4 8}$ & $\mathbf{3 . 6 4 4}$ \\
10 & 136 & HOXA2 & $<1 \mathrm{e}-07$ & $<1 \mathrm{e}-07$ & 1.402 & 2.704 \\
\hline
\end{tabular}


A

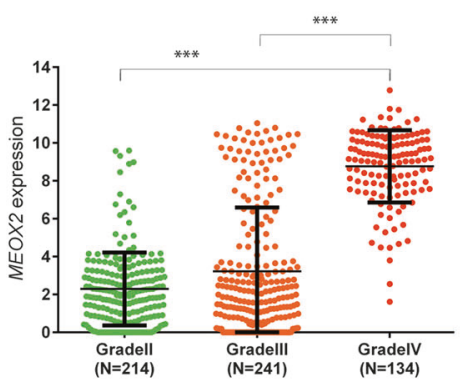

B

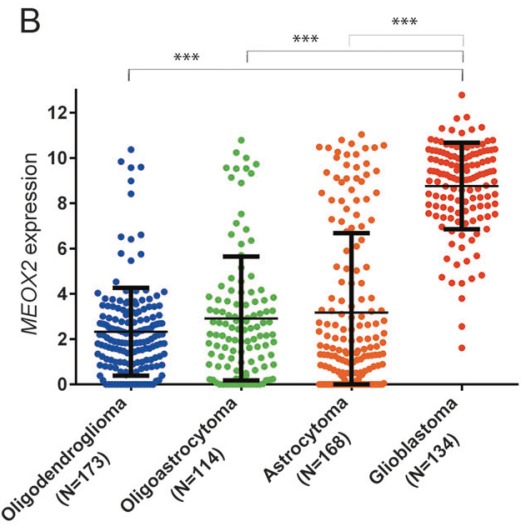

C

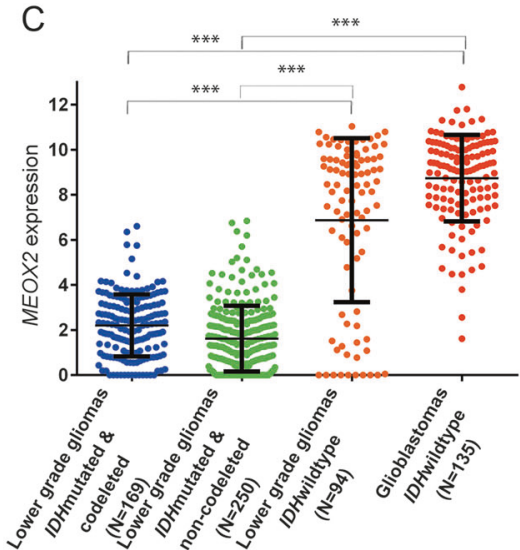

D

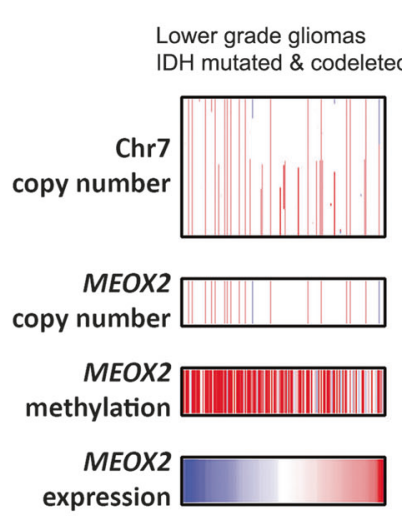

Lower grade gliomas IDH mutated \& non-codeleted

Lower grade gliomas IDH Glioblastoma wildtype

IDH wildtype
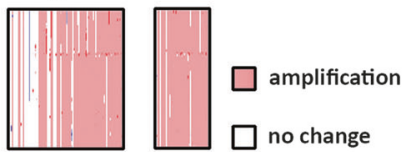

deletion
E

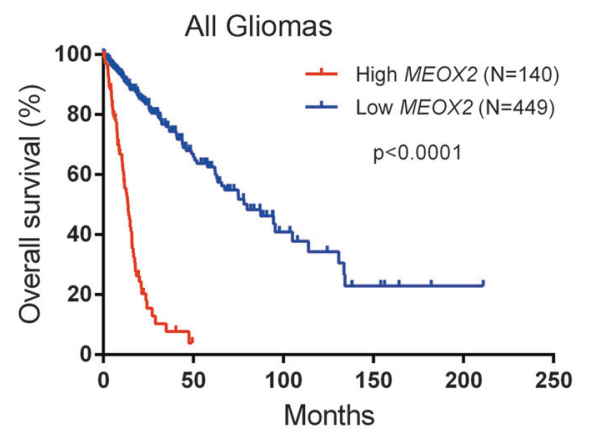

G

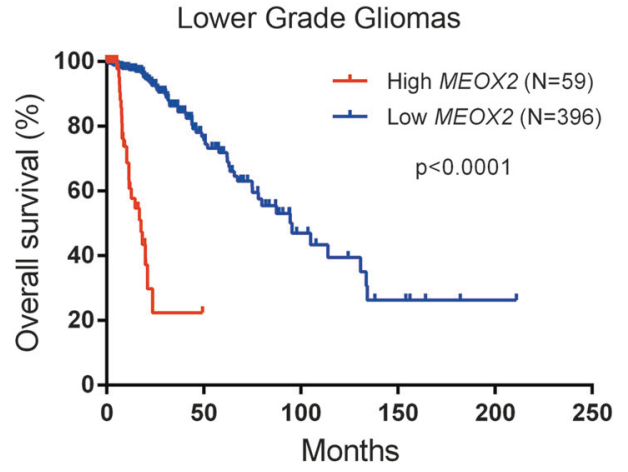

$\mathrm{F}$

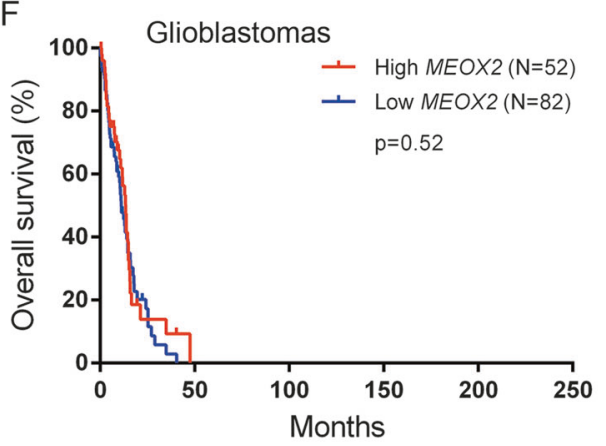

H Lower Grade Gliomas IDH1/2 wildtype

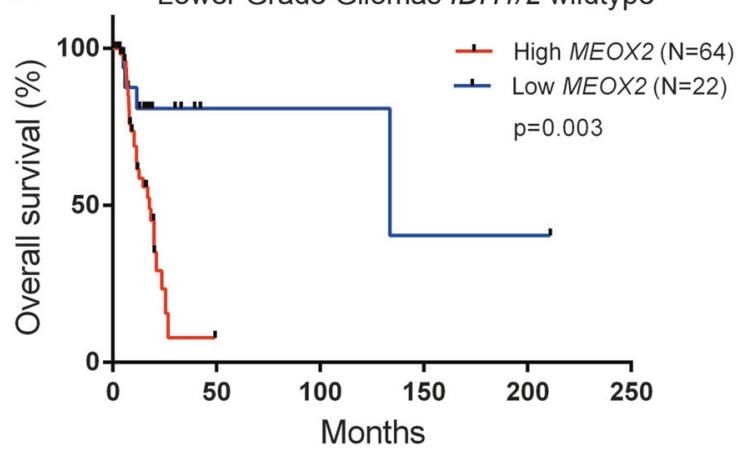

contrary to lower grade glioma cohort (Fig. 1g) (p $<$ $0.0001), M E O X 2$ mRNA was not associated with poor overall survival in glioblastomas (Fig. 1f). Focusing on lower grade gliomas, we performed survival analysis on 
Fig. 1 Expression and clinical relevance of $M E O X 2$ in TCGA database (a, b, c; Log2). Dot plot representing MEOX2 expression in TCGA according to histoprognostic grade (a), histological type (WHO 2007) (b), and WHO 2016 molecular classification (c). (d) Heat map of GISTIC2 thresholded copy number calls in chr7 and MEOX2, methylation status (beta-value) and expression (RSEM $\log 2$ ) of MEOX2 according to glioma molecular subtypes. (e-h) Kaplan-Meier curves plotting overall survival in all gliomas (e), glioblastomas (f), lower grade gliomas (g) and in lower grade gliomas $I D H 1 / 2$ wildtype (h). Kruskal-Wallis test and Mantel-Cox log rank test were performed to determine the p-value indicated on the panels

each molecular subtype according to the MEOX2 mRNA mean level and found no significant results (Figure S4). However, we noticed a subgroup in $I D H 1 / 2$ wildtype tumors corresponding to the first quartile with a very low MEOX2 mRNA level similar to IDH1/2 mutated samples, whereas the remaining major fraction had MEOX2 mRNA at the same level as glioblastomas (Fig. 1c). More interestingly, this lower quartile of $I D H 1 / 2$ wildtype lower grade gliomas showed better overall survival $(\mathrm{p}=0.003$, Fig. 1h). In this subgroup, univariate and multivariate analysis including clinicopathological and molecular features showed that $M E O X 2$ was an independent prognosis marker ( $\mathrm{p}=0.007$ and 0.017 respectively) (Table 3). Interestingly, MEOX2 mRNA level predicted overall survival more accurately than Chr7gain/Chr10loss, TERT expression, ATRX mutational status or MGMT methylation.

\section{MEOX2 associations confirmed in other public sets CGGA and REMBRANDT}

To further confirm our disclosures, we analyzed the results of RNAseq data of 273 tumors from the CGGA dataset and microarray data of 311 gliomas from the REMBRANDT cohort. In both cohorts, we found a positive correlation between MEOX2 mRNA abundance and tumor grade $(\mathrm{p}<$ 0.0001, Figure S5A, Figure S6A). As in TCGA cohort, IDH1 wildtype gliomas highly expressed $M E O X 2$ in comparison with IDH1 mutated gliomas $(\mathrm{p}<0.0001$, Figure S5B) and its mRNA expression negatively correlated with overall survival in both CGGA and Rembrandt cohorts $(\mathrm{p}<0.0001$ Figure S5C and Figures S6B). Finally, MEOX2 mRNA level negatively correlated with survival in lower grade gliomas $(p<0.0001)$, barely or not in glioblastomas, thereby corroborating the previous results showing $M E O X 2$ as an important marker of progression in lower grade gliomas (Figure S5D, E and $\mathrm{S} 6 \mathrm{C}, \mathrm{D})$. Unlike TCGA, the prognostic impact of $M E O X 2$ in IDH1 wildtype lower grade gliomas cohort was not significant, probably due to the small number of samples $(\mathrm{n}=49)$ (Figure S5F).

\section{Immunohistochemistry investigation confirmed MEOX2 and IDH1 wildtype glioma association}

To further explore the previous findings, clinical and biological data and tumor samples from 112 patients with gliomas were collected. MEOX2 immunohistochemistry analysis on tumor samples transferred into tissue microarrays was carried out. Immunohistochemistry analysis showed the nuclear localization of MEOX2 protein, which was in coherence with its transcription factor function (Fig. 2a). Scoring analysis showed that the highest expression of MEOX2 protein was observed in IDH1 wildtype gliomas (88\% glioblastomas, $11 \%$ lower grade gliomas) whereas IDHI mutated (100\% lower grade gliomas) remained poorly or negatively stained $(p=0.0152$, Fig. $2 b)$. Combined lower grade gliomas and glioblastomas were consequently separated into two groups according to IDHI mutational status. We analyzed the distribution of negative and positive MEOX2 samples according to IDH1 mutational profile and found that $I D H 1$ wildtype gliomas comprised $80 \%$ of samples with positive MEOX2 staining (Fig. 2c). Among IDH1 wildtype, $100 \%$ of the lower grade gliomas and $77 \%$ of glioblastomas were positive for MEOX2 staining. To confirm the prominent distribution of MEOX2 into the most aggressive molecular tumor subtypes, the $I D H I$ wildtype tumors, we looked back into the independent cohort dataset. The association between MEOX2 mRNA expression and aggressive glioma subtypes was even clearer in TCGA dataset. Indeed, only $I D H 1 / 2$ wildtype gliomas, comprising 59\% glioblastomas and $41 \%$ lower grade gliomas, highly expressed MEOX2 mRNA (Fig. 2d) whereas no $I D H 1 / 2$ mutated gliomas did so.

\section{MEOX2 protein expression revealed a prognostic factor in lower grade gliomas}

Kaplan-Meier survival analysis of the clinical cohort revealed that positive MEOX2 staining was associated with poor overall survival $(\mathrm{p}=0.009)$ and progression-free survival $(p=0.0078)$ (Fig. 3a, b). The same association was observed while analyzing only the lower grade glioma cohort with $\mathrm{p}=0.027$ and $\mathrm{p}=0.078$ for overall survival and progression-free survival respectively, suggesting that MEOX2 could be an interesting prognostic factor (Fig. 3c, d). However, no association was found in glioblastoma for overall survival and progression-free survival (Fig. 3e, f).

Finally, we performed univariate and multivariate analysis including clinicopathological and molecular features. Univariate Cox regression analysis showed an association between MEOX2 protein expression and overall survival $(\mathrm{p}=0.012)$ and progression-free survival $(\mathrm{p}=0.009)$ in all gliomas (Table 4). In lower grade gliomas, MEOX2 tended 
Table 3 Univariate and multivariate analysis of lower grade IDHI/2 wildtype gliomas of the TCGA dataset (NA: not analyzed, CI: confidence interval)

\begin{tabular}{|c|c|c|c|c|c|c|}
\hline \multirow{2}{*}{$\begin{array}{l}\text { Lower grade } I D H I / 2 \text { wildtype } \\
\text { gliomas }(\mathrm{n}=86) \text { Variables }\end{array}$} & \multirow[t]{2}{*}{ Data description } & & \multicolumn{4}{|l|}{ Overall survival } \\
\hline & & & $\begin{array}{l}\text { Univariate analysis } \\
\text { Hazard ratio (CI 95\%) }\end{array}$ & p-value & $\begin{array}{l}\text { Multivariate analysis } \\
\text { Hazard ratio (CI 95\%) }\end{array}$ & p-value \\
\hline Age & Mean & 51.6 & $1.056(1.025-1.088)$ & $<0.0001$ & $1.054(1.021-1.087)$ & .001 \\
\hline \multirow[t]{2}{*}{ Gender } & Female & 39 & $0.749(0.366-1.533)$ & 0.428 & & \\
\hline & Male & 47 & & & & \\
\hline \multirow[t]{2}{*}{ MGMT promoter status } & Methylated & 31 & $0.666(0.322-1.377)$ & 0.272 & & \\
\hline & Unmethylated & 55 & & & & \\
\hline \multirow[t]{3}{*}{ Chr 7 gain/Chr 10 loss } & Yes & 47 & $0.644(0.308-1.346)$ & 0.242 & & \\
\hline & No & 38 & & & & \\
\hline & NA & 1 & & & & \\
\hline \multirow[t]{3}{*}{ Chr 19/20 co-gain } & Yes & 75 & $0.795(0.278-2.276)$ & 0.669 & & \\
\hline & No & 10 & & & & \\
\hline & NA & 1 & & & & \\
\hline \multirow[t]{3}{*}{ TERT expression status $(\log (2)>2)$} & Yes & 52 & $0.462(0.21-1.016)$ & 0.055 & & \\
\hline & No & 33 & & & & \\
\hline & NA & 1 & & & & \\
\hline \multirow[t]{2}{*}{$A T R X$ status } & Mutant & 7 & $1.055(0.359-3.1)$ & 0.923 & & \\
\hline & Wildtype & 79 & & & & \\
\hline \multirow[t]{2}{*}{ MEOX2 expression (lower quartile) } & High & 64 & $0.187(0.055-0.634)$ & 0.007 & $0.222(0.065-0.761)$ & 0.017 \\
\hline & Low & 22 & & & & \\
\hline
\end{tabular}

to be associated with overall survival $(\mathrm{p}=0.058)$ and progression-free survival ( $\mathrm{p}=0.069)$, a finding corroborating survival analysis outcomes (Table 5). Taken together, these results suggested MEOX2 as an interesting prognostic marker in gliomas.

\section{MEOX2 involved in several major carcinogenesis pathways}

As $M E O X 2$ was mainly up-regulated in glioblastoma, we explored its expression according to Verhaak's classification. In TCGA dataset, a significant increase of MEOX2 mRNA was observed in the classical subtype in comparison with proneural $(\mathrm{p}<0.0001)$, mesenchymal $(\mathrm{p}<0.001)$ and neural subtypes $(\mathrm{p}<0.01)$ (Fig. 4a). MEOX2 mRNA enrichment in the classic subtype was even clearer in the CGGA dataset (Fig. 4b). Additionally, we explored MEOX2 mRNA expression over distinct regions of the tumor from the Ivy Glioblastoma Atlas dataset [22]. MEOX2 mRNA level was significantly higher in tumor cells and infiltrating tumors than in other components of the tumor such as the perinecrotic zone, proliferating vessels or leading edge (Fig. 4c).

We then analyzed the positively and negatively impacted genes associated with $M E O X 2$ expression in glioblastoma. As expected, we found that marker genes of classical subtype signaling such as Notch (JAG1, LFNG) and Sonic Hedgehog (SMO, GASI and GLI2) were mostly positively regulated in MEOX2 expression network (Figure S7, Table S1).
Finally, we wished to determine in which biological process $M E O X 2$ was mainly implicated. By performing GSEA analysis on glioblastoma RNAseq data from TCGA using C5.p.v6.1 gene set from the Molecular Signatures Database (MSigDB), several major Go terms appeared (Fig. 4d), all of them involved in replication, recombination and mitosis. Interestingly, NOS2, an induced marker in neurosphere glioma cell lines, was highly enriched in glioblastomas with an elevated MEOX2 mRNA level (Table S2) [23].

\section{Discussion}

Glioma is the most common type of intracranial primary tumor comprising different subtypes of which glioblastoma is the most malignant. Based on the previous histopathological classification system, there was a high rate of intra/ interobserver variability leading to divergent diagnoses and inexact prognostic outcomes [4, 5]. Fortunately, in May 2016, the latest version of the 2016 WHO Classification was published, providing more accurate stratification than classification based solely on histopathology [6]. Indeed, it introduced molecular markers, i.e. IDH1/2 mutational status and $1 \mathrm{p} / 19 \mathrm{q}$ codeletion, which are recognized worldwide for their high predictive value [24]. Despite the extended time of molecular investigation of glioma profile, to our knowledge MEOX2 has never been reported even though we have demonstrated that it is an interesting prognostic marker. 

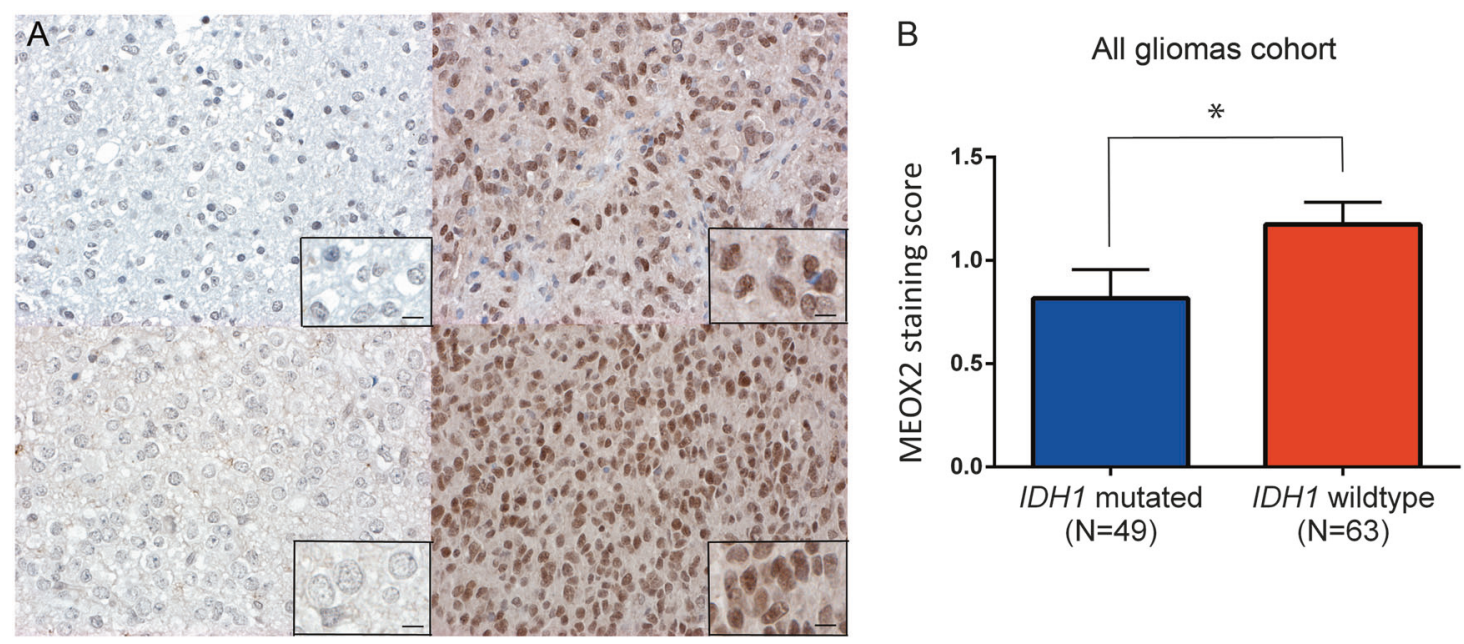

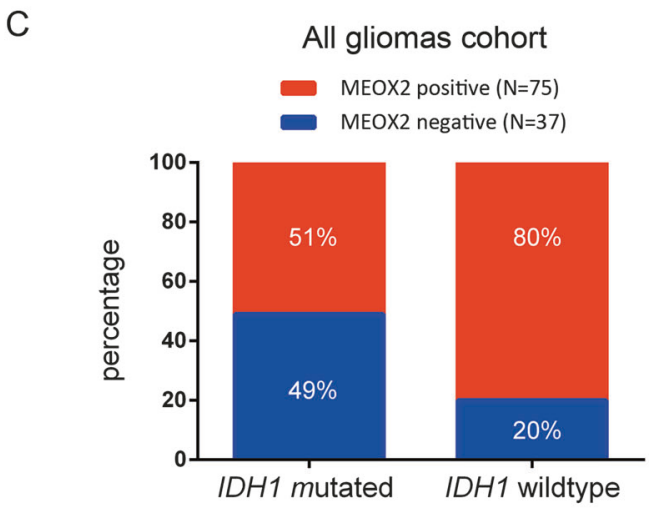

Fig. 2 MEOX2 expression is related to $I D H 1$ molecular subtypes in TCGA and in clinical data sets. (a) Immunohistochemical analysis of MEOX2 protein expression according to IDH1 mutational status showing the strong nuclear location of MEOX2 in IDH1 wildtype tumors (right panels (Scale bar: $10 \mu \mathrm{m}$ ). (b) Histogram representing the MEOX2 staining scores according to $I D H 1$ status in the clinical cohort $(\mathrm{n}=112,56$ glioblastomas and 56 lower grade gliomas). Histograms

In this study, we identified a new transcription factor of interest in glioma, MEOX2. We showed that MEOX2 is correlated with $I D H 1 / 2$ mutational status in public datasets and local clinical datasets. We demonstrated that MEOX2 is a potent prognostic factor of patient outcome in all gliomas and in lower grade gliomas alone. Moreover, it appeared to be a robust prognostic marker of survival in the $I D H 1 / 2$ wildtype lower grade glioma subpopulation, independent of the combination of chr7 gain/chr10 loss. Finally, we highlighted replication, recombination and mitosis pathways positively correlated with MEOX2 up-regulation in glioblastoma.

Several reports have suggested that high expression of homeotic genes, HOXA9 [25], HOXD4 [26] and HOXA13 [27] was an indicator of poor prognosis in glioblastoma patients. We corroborated literature data showing that the ten major transcription factors highly correlated with patient overall survival in gliomas mostly belonged to HOX family. However, the transcription factor MEOX2 which was not a classical HOX family member, has seldom been reported in gliomas, and remains ranked ninth.

MEOX2 is a homeobox protein, a mesodermal transcription factor that plays a key role in somites and limb genesis [28]. In non-tumor tissue, MEOX2 has been described as a cell cycle inhibitor through CDKN1A and CDKN2A activation in endothelial cells [7, 29]. Therefore, it has been described mainly as a negative regulator of angiogenesis and cell proliferation [8]. In tumor tissue, the role of MEOX2 is not clear. MEOX2 has been reported as a tumor suppressor gene in Wilms syndrome, hepatocellular carcinoma and larynx carcinoma, in which MEOX2 loss has been correlated with shorter overall survival and poor 
A

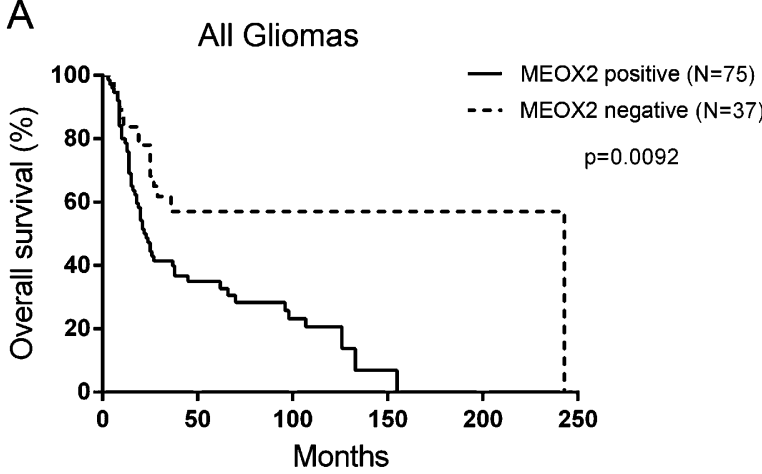

C

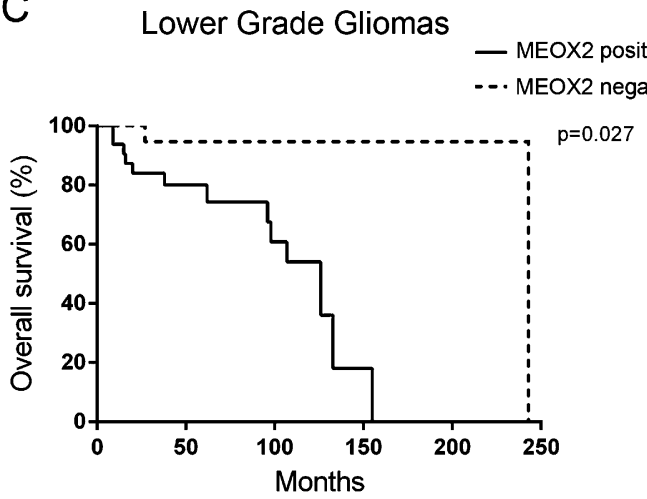

$E$

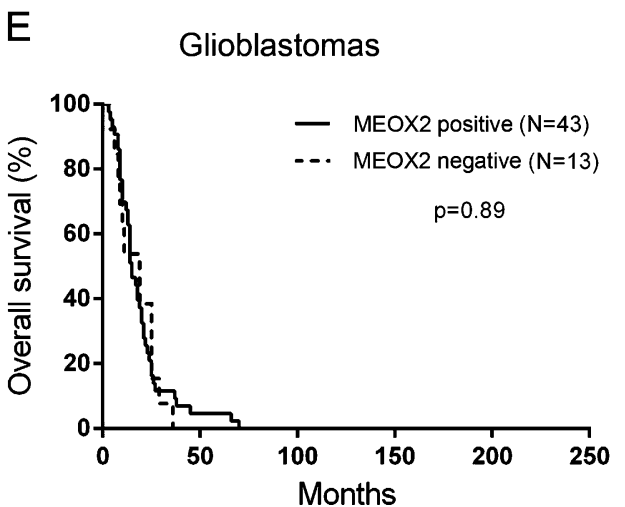

Fig. 3 MEOX2 is associated with poor glioma patient outcome in clinical cohort. (a-f) Kaplan-Meier survival curves plotting overall survival or progression-free survival according to MEOX2 staining in

disease-free survival $[12,14,15]$. However, MEOX2 overexpression has been associated with chemoresistance and poor prognosis factor in lung carcinoma [13]. In glioma, conflicting data have also been reported regarding MEOX2 expression. Bao et al. found a signature of 17 genes including $M E O X 2$, which correlated with overall survival in a cohort of mesenchymal glioblastomas [18]. Conversely, Vastrad et al. reported down-regulation of MEOX2 in various cells compared to normal astrocytes [19]. However, the 15 cell types studied were of diverse origins: glioma stem

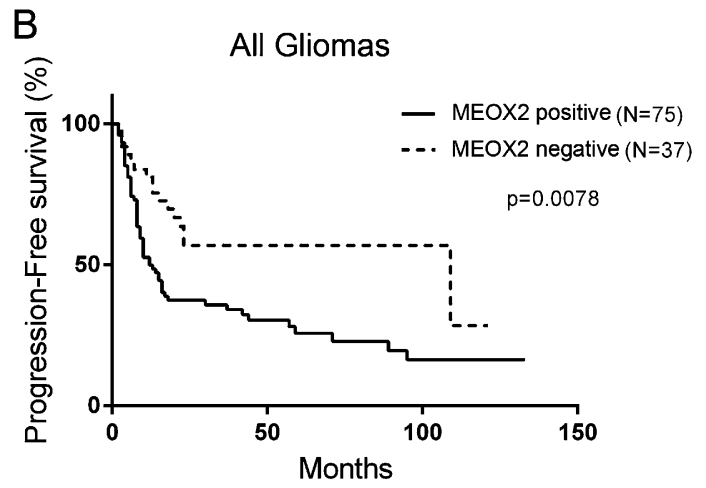

D Lower Grade Gliomas
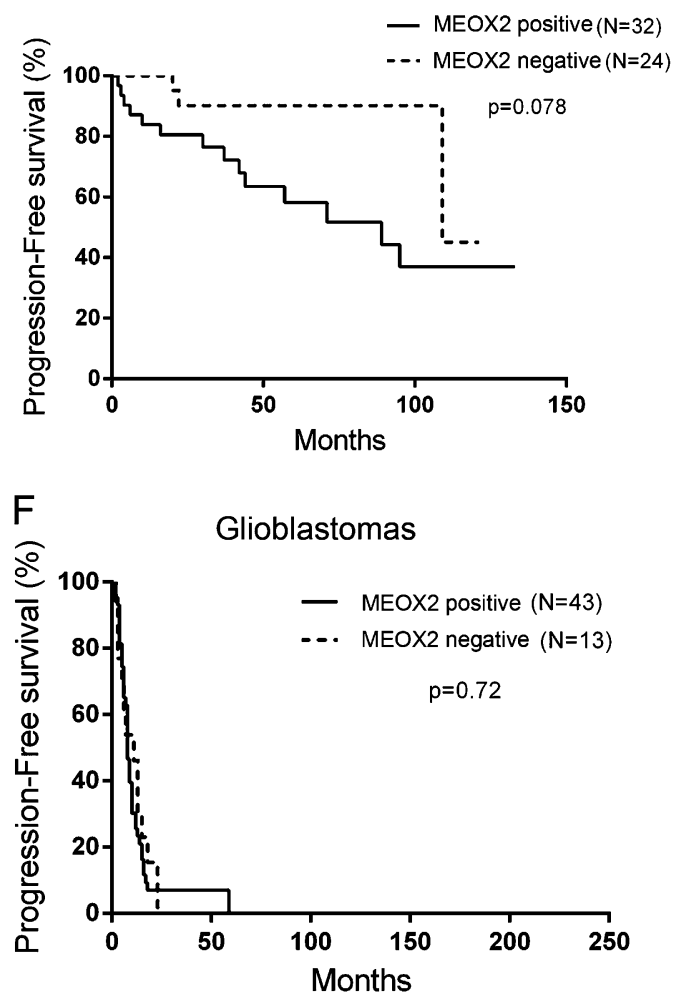

all gliomas (a, b), lower grade gliomas (c, d) and glioblastomas (e, f). Mantel-Cox log rank test was performed to determine the p-value indicated on the graphs

cell lines, astrocytes overexpressing oncogenic and iPSCinducing factors and glioblastoma conventional cell lines, and considering them as a group is controversial. None of these studies correlated MEOX2 expression with glioma molecular profile, either in glioblastomas or in lower grade gliomas. For the first time we showed in several independent cohorts that MEOX2, mRNA and protein, is negatively correlated with progression-free survival and overall survival in gliomas and in lower grade gliomas. Additionally, we demonstrated that MEOX2 was associated with $I D H 1 / 2$ 


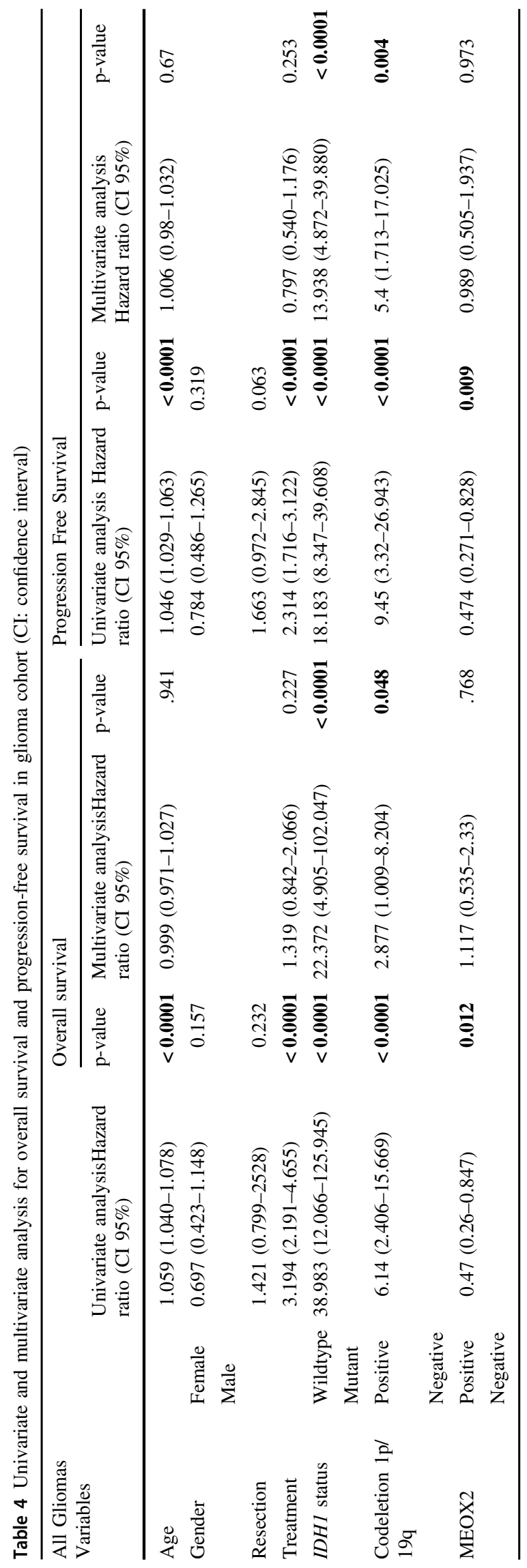

wildtype, a known molecular marker of aggressiveness. Consequently, MEOX2 expression should be considered when assessing the prognosis value of gliomas, particularly lower grade gliomas.

While MEOX2 has been described as an antiangiogenic factor, its function in gliomagenesis is unknown. According to Ivy dataset, MEOX2 mRNA is confined to tumor cells and tumor infiltrating rather than vascular tissue. Moreover, GSEA analysis supported the idea that MEOX2 was mainly involved in proliferation, replication and mitosis biological processes. This contrasted with MEOX2 physiological functions in cell cycle arrest [7,29] and vascular endothelial growth arrest control via NFkB inhibition [11]. The dual function of members of homeobox family has been previously described; they activate growth and migration to promote angiogenesis on the one hand, and to restore or maintain quiescent state on the other hand [30]. Therefore, regarding our results, it seems that the MEOX2 pathways at stake in gliomas are different from the pathways previously described in literature in normal endothelial cells or in hepatocarcinoma tissues.

MEOX2 mRNA was enriched in classical Verhaak subtype and correlated with a chromosome 7 gain and a poor methylation profile. Copy number alteration and decrease of methylation prints at MEOX2 locus are two mechanisms that could explain, at least to some extent, the elevated level of MEOX2 mRNA and MEOX2 protein observed in glioblastoma. Interestingly, $C D K N 2 A$, a known MEOX2 target in endothelial cells, is frequently homologously deleted in the classical subclass, which corroborated the assumption that MEOX2 targeted different genes in glioblastomas than in endothelial cells [31].

One of the upstream effectors that may be responsible for the difference in MEOX2 mRNA level between the molecular subtypes of glioma could be the IDH1 itself. Indeed, it has been demonstrated that IDH1 R132H mutation induced persistent down-regulation of MEOX2 in immortalized human astrocytes (IHAs) and patient-derived glioma tumorspheres [32]. Little is known about other possible MEOX2 regulators. In HUVEC (Human Umbilical Vein Endothelial Cell), microRNA-221 upregulated MEOX2 through ZEB2 activation, a zinc finger nuclear factor [33]. In human hepatocellular carcinoma and lung adenocarcinoma, down-regulation of microRNA-301 has been shown to be responsible for $M E O X 2$ activation [34, 35]. To our knowledge, no specific molecule has ever been designed to target MEOX2.

To conclude, our work highlighted a new relevant molecular biomarker in glioma. Further explorations will be needed to define the position of MEOX2 in gliomagenesis and to establish the exact mechanisms responsible for $M E O X 2$ up-regulation in $I D H 1 / 2$ wildtype gliomas. 


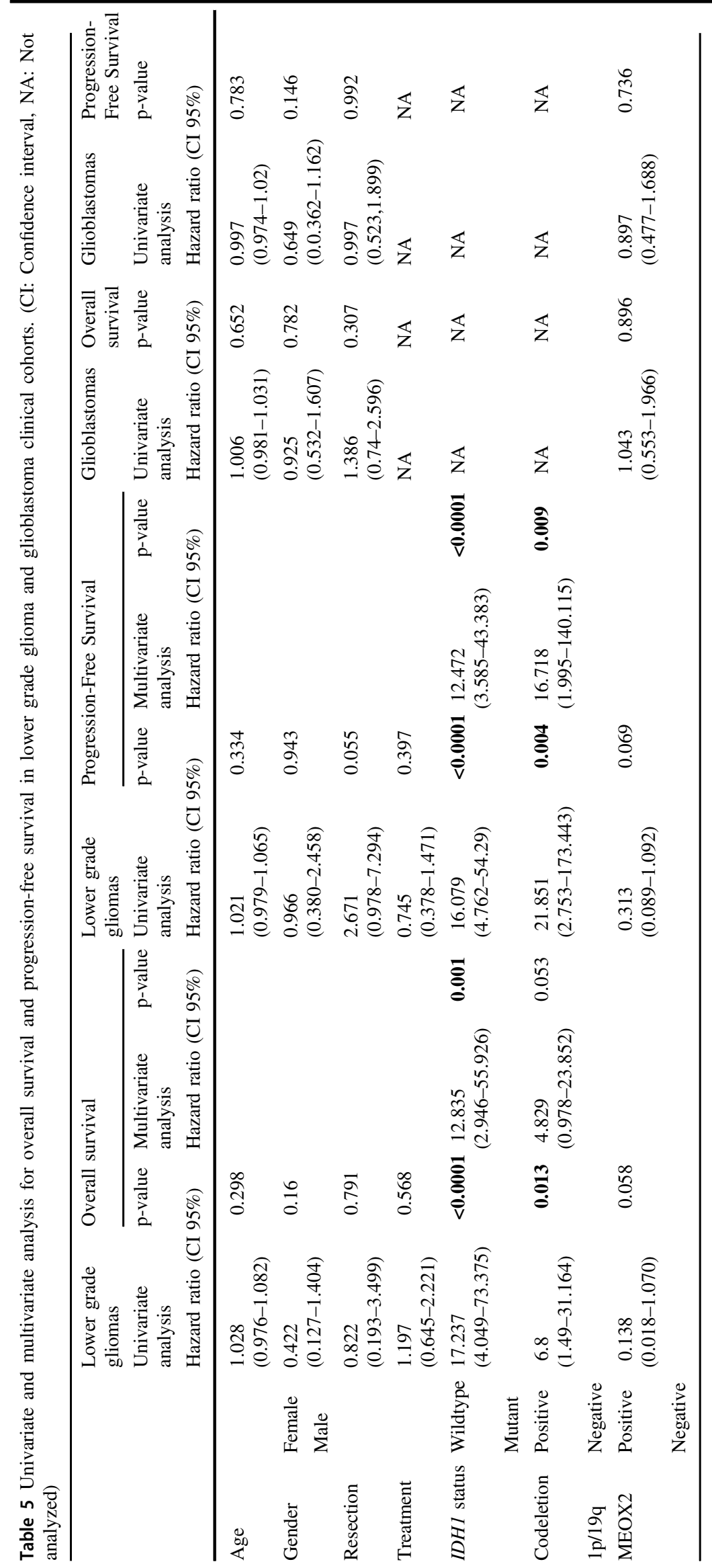



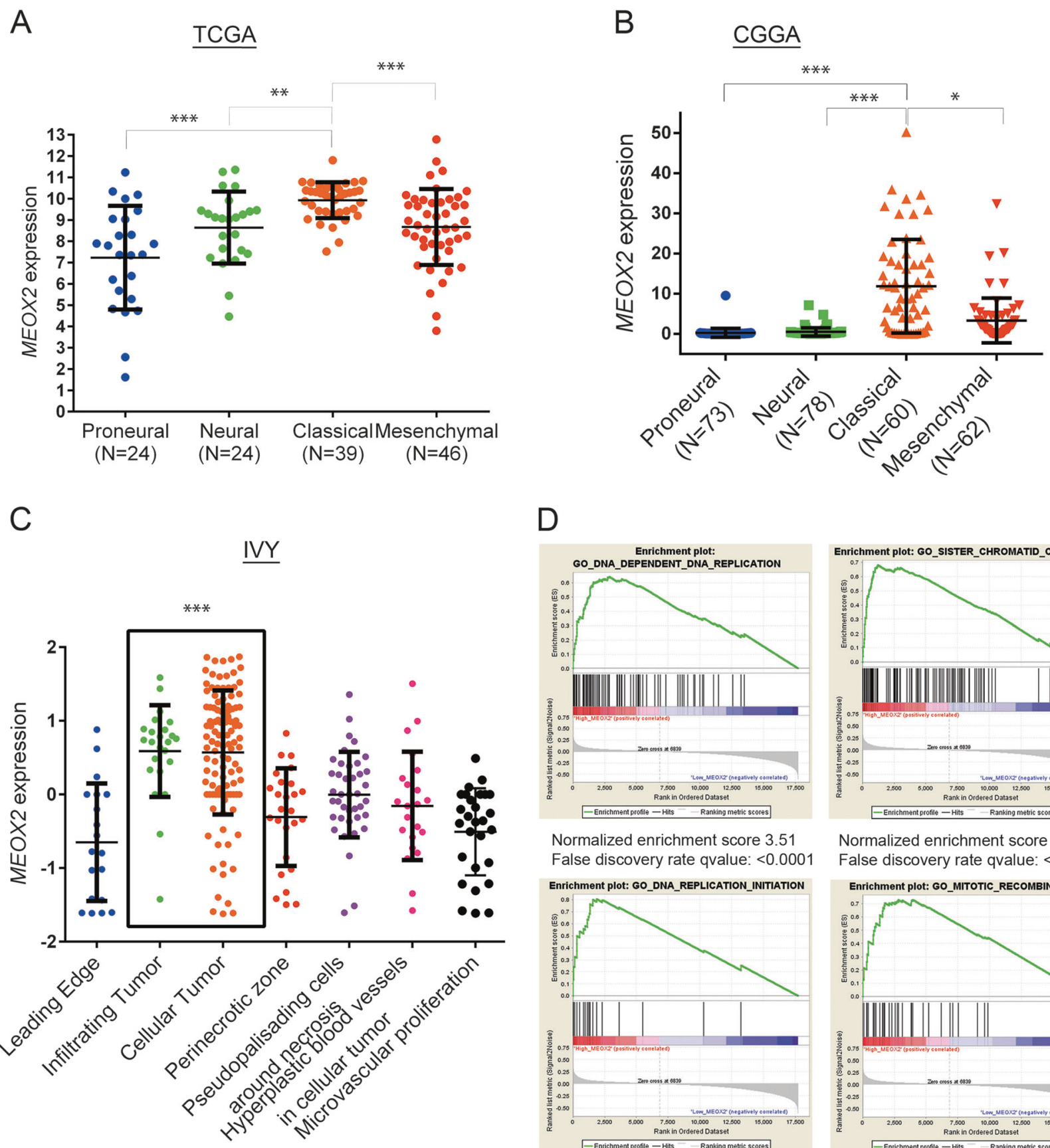

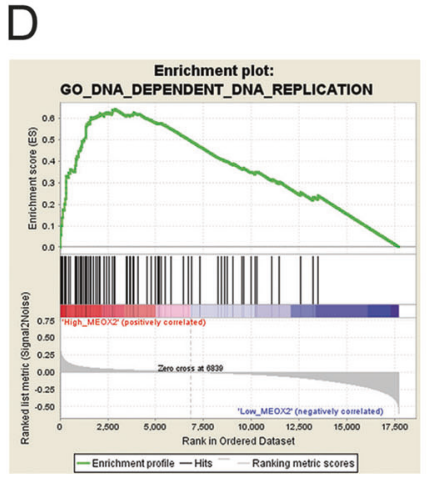

Normalized enrichment score 3.51

False discovery rate qvalue: $<0.0001$

Enrichment plot: GO_DNA_REPLICATION_INITIATION

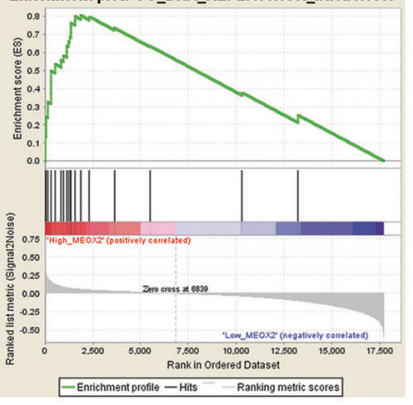

Normalized enrichment score 2.83

False discovery rate qvalue: $<0.0001$

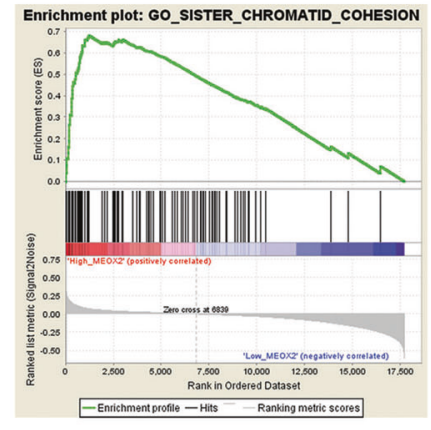

Normalized enrichment score 2.88

False discovery rate qvalue: $<0.0001$

Enrichment plot: GO_MITOTIC_RECOMBINATION

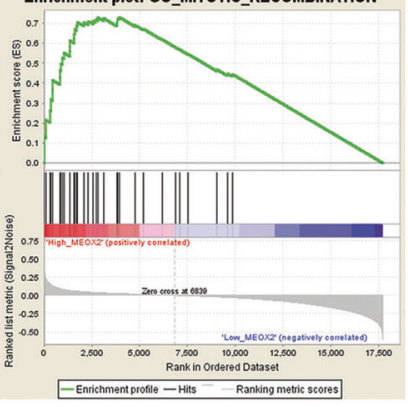

Normalized enrichment score 2.74

False discovery rate qvalue: $<0.0001$

Fig. 4 Major pathways and biological relevance of MEOX2 in glioblastoma. (a, b) Dot Plot showing MEOX2 mRNA expression in the proneural, neural, classical and mesenchymal subtypes of glioblastomas from TCGA (a) and CGGA (b) datasets. (c) Dot Plot showing MEOX2 mRNA abundance over distinct regions of the

Acknowledgements This work was supported by the Ligue contre le Cancer of Grand Ouest from the comity of Vienne and Deux-Sèvres departments, and by "En avant la vie" association. The authors wish to thank Jeffrey Arsham, an American translator, for having reviewed and revised the original English-language text.

tumors from IVY glioblastoma dataset. Kruskal-Wallis test was performed to determine statistical significance. (d) The four most illustrative signatures after GSEA analysis in TCGA glioblastoma cohort with Normalized Enrichment Score $\geq 2.74$ and False Discovery Rate $<$ 0.0001

\section{Compliance with ethical standards}

Conflict of interest The authors declare that they have no conflict of interest. 
Publisher's note: Springer Nature remains neutral with regard to jurisdictional claims in published maps and institutional affiliations.

\section{References}

1. Omuro A, DeAngelis LM. Glioblastoma and other malignant gliomas: a clinical review. JAMA. 2013;310:1842-50.

2. Stupp R, Hegi ME, Mason WP, et al. Effects of radiotherapy with concomitant and adjuvant temozolomide versus radiotherapy alone on survival in glioblastoma in a randomised phase III study: 5-year analysis of the EORTC-NCIC trial. Lancet Oncol. 2009;10:459-66.

3. Louis DN, Ohgaki H, Wiestler OD, et al. The2007 WHO Classification of Tumours of the Central Nervous System. Acta Neuropathol (Berl). 2007;114:97-109.

4. Aldape K, Simmons ML, Davis RL, et al. Discrepancies in diagnoses of neuroepithelial neoplasms: the San Francisco Bay Area Adult Glioma Study. Cancer. 2000;88:2342-9.

5. van den Bent MJ. Interobserver variation of the histopathological diagnosis in clinical trials on glioma: a clinician's perspective. Acta Neuropathol (Berl). 2010;120:297-304.

6. Louis DN, Perry A, Reifenberger G, et al. The 2016 World Health Organization Classification of Tumors of the Central Nervous System: a summary. Acta Neuropathol (Berl). 2016;131:803-20.

7. Douville JM, Cheung DYC, Herbert KL, et al. Mechanisms of MEOX1 and MEOX2 regulation of the cyclin dependent kinase inhibitors p21 and p16 in vascular endothelial cells. PLoS ONE. 2011;6:e29099.

8. Gorski DH, Leal AJ. Inhibition of endothelial cell activation by the homeobox gene Gax. J Surg Res. 2003;111:91-99.

9. Fisher SA, Siwik E, Branellec D, et al. Forced expression of the homeodomain protein Gax inhibits cardiomyocyte proliferation and perturbs heart morphogenesis. Development. 1997;124: 4405-13.

10. Zheng $\mathrm{H}, \mathrm{Hu} \mathrm{Z}$, Zhai $\mathrm{X}$, et al. Gax regulates human vascular smooth muscle cell phenotypic modulation and vascular remodeling. Am J Transl Res. 2016;8:2912-25.

11. Patel S, Leal AD, Gorski DH. The Homeobox Gene Gax Inhibits Angiogenesis through Inhibition of Nuclear Factor- $\mathrm{kB}-$ Dependent Endothelial Cell Gene Expression. Cancer Res. 2005;65:1414-24.

12. Zhou P, Chen Z, Chang R-M, et al. Growth arrest-specific homeobox is associated with poor survival in patients with hepatocellular carcinoma. Med Oncol. 2012;29:3063-9.

13. Ávila-Moreno F, Armas-López L, Álvarez-Moran AM, et al. Overexpression of MEOX2 and TWIST1 is associated with H3K27me3 levels and determines lung cancer chemoresistance and prognosis. PLoS ONE. 2014;9:e114104.

14. Tian L, Tao ZZ, Ye HP, et al. Over-expression of MEOX2 promotes apoptosis through inhibiting PI3K/Akt pathway in laryngeal cancer cells. Neoplasma 2018. https://doi.org/10.4149/neo_2018_ $171218 \mathrm{~N} 824$

15. Ohshima J, Haruta M, Arai Y, et al. Two candidate tumor suppressor genes, MEOX2 and SOSTDC1, identified in a $7 \mathrm{p} 21$ homozygous deletion region in a Wilms tumor. Genes Chromosomes Cancer. 2009;48:1037-50.

16. Armas-López L, Piña-Sánchez P, Arrieta O, et al. Epigenomic study identifies a novel mesenchyme homeobox2-GLI1 transcription axis involved in cancer drug resistance, overall survival and therapy prognosis in lung cancer patients. Oncotarget. 2017;8:67056.

17. Verhaak RGW, Hoadley KA, Purdom E, et al. An integrated genomic analysis identifies clinically relevant subtypes of glioblastoma characterized by abnormalities in PDGFRA, IDH1, EGFR and NF1. Cancer Cell. 2010;17:98.

18. Bao Z-S, Zhang C-B, Wang H-J, et al. Whole-Genome mRNA Expression Profiling Identifies Functional and Prognostic Signatures in Patients with Mesenchymal Glioblastoma Multiforme. CNS Neurosci Ther. 2013;19:714-20.

19. Vastrad B, Vastrad C, Godavarthi A, et al. Molecular mechanisms underlying gliomas and glioblastoma pathogenesis revealed by bioinformatics analysis of microarray data. Med Oncol North Lond Engl. 2017;34:182.

20. Ohgaki H, Kleihues P. The definition of primary and secondary glioblastoma. Clin Cancer Res J Am Assoc Cancer Res. 2013;19:764-72.

21. Nobusawa S, Watanabe T, Kleihues P, et al. IDH1 Mutations as Molecular Signature and Predictive Factor of Secondary Glioblastomas. Clin Cancer Res. 2009;15:6002-7.

22. Cantanhede IG, Oliveira JRM de. PDGF Family Expression in Glioblastoma Multiforme: Data Compilation from Ivy Glioblastoma Atlas Project Database. Sci Rep 2017;7. https://doi.org/ 10.1038/s41598-017-15045-w.

23. Palumbo P, Miconi G, Cinque B, et al. NOS2 expression in glioma cell lines and glioma primary cell cultures: correlation with neurosphere generation and SOX-2 expression. Oncotarget. 2017;8:25582-98.

24. Kim Y-H, Nobusawa S, Mittelbronn M, et al. Molecular Classification of Low-Grade Diffuse Gliomas. Am J Pathol. 2010;177:2708-14.

25. Costa BM, Smith JS, Chen Y, et al. Reversing HOXA9 oncogene activation by PI3K inhibition: epigenetic mechanism and prognostic significance in human glioblastoma. Cancer Res. 2010;70:453-62.

26. Zhao X-W, Zhan Y-B, Bao J-J, et al. Clinicopathological analysis of HOXD4 expression in diffuse gliomas and its correlation withIDHmutations and $1 \mathrm{p} / 19 \mathrm{q}$ co-deletion. Oncotarget. 2017;8:115657-66.

27. Duan R, Han L, Wang Q, et al. HOXA13 is a potential GBM diagnostic marker and promotes glioma invasion by activating the Wnt and TGF- $\beta$ pathways. Oncotarget. 2015;6:27778-93.

28. Mankoo BS, Skuntz S, Harrigan I, et al. The concerted action of Meox homeobox genes is required upstream of genetic pathways essential for the formation, patterning and differentiation of somites. Dev Camb Engl. 2003;130:4655-64.

29. Irelan JT, Gutierrez Del Arroyo A, Gutierrez A, et al. A functional screen for regulators of CKDN2A reveals MEOX2 as a transcriptional activator of INK4a. PLoS One. 2009;4:e5067.

30. Kachgal S, Mace KA, Boudreau NJ. The dual roles of homeobox genes in vascularization and wound healing. Cell Adhes Migr. 2012;6:457-70.

31. Goodenberger ML, Jenkins RB. Genetics of adult glioma. Cancer Genet. 2012;205:613-21

32. Turcan S, Makarov V, Taranda J, et al. Mutant-IDH1-dependent chromatin state reprogramming, reversibility, and persistence. Nat Genet. 2018;50:62.

33. Chen Y, Banda M, Speyer CL, et al. Regulation of the expression and activity of the antiangiogenic homeobox gene GAX/MEOX2 by ZEB2 and microRNA-221. Mol Cell Biol. 2010;30:3902-13.

34. Cao G, Huang B, Liu Z, et al. Intronic miR-301 feedback regulates its host gene, ska2, in A549 cells by targeting MEOX2 to affect ERK/CREB pathways. Biochem Biophys Res Commun. 2010;396:978-82.

35. Zhou P, Jiang W, Wu L, et al. miR-301a Is a Candidate Oncogene that Targets the Homeobox Gene Gax in Human Hepatocellular Carcinoma. Dig Dis Sci. 2012;57:1171-80. 